\title{
Past, Present and Future of Quantum Computing: A Systematic Study
}

\author{
Palash Dutta Banik, Asoke Nath \\ Department of Computer Science, St. Xavier's College (Autonomous), Kolkata, India
}

\begin{abstract}
Article Info

Volume 7, Issue 6

Page Number : 351-363

Publication Issue :

November-December-2021

Article History

Accepted : 12 Dec 2021

Published : 26 Dec 2021

Quantum Computing is relatively new and it's kind of a special type of computing that uses the laws of quantum physics. In classical computing, we have some limitations and we can overcome those with the help of Quantum Computing as it uses qubits, but we need to keep those qubits at low temperature. Quantum Computing uses the probabilistic nature of electrons. The power of a quantum computer increases exponentially with the number of qubits linked tougher.

Quantum computers are very difficult to make but there are a huge number of calculations that can be done easily with the use of a quantum computer. Quantum computers are way better and faster than classical computers. So, we can say that quantum computers rather than quantum computing will be used in the near future to replace classical computing.

Keywords : Quantum Computing, Classical Computing, Super Computer, Highperformance computer, Superposition, Entanglement, Quantum Computer Experiment.
\end{abstract}

\section{INTRODUCTION}

In the year 1927, 29 scientists gather in Brussels to discuss physics. Those few days, they wrestled with the question of quantum determinacy and whether our world at the minutest level operates as a fixed system or merely as a group of probabilities. They discuss the problem of light. For nearly three centuries since Newton wrote his famous treatise on optics, physicists had debated whether light was a particle or a wave. At first, Newton told, that light is a rain of particles. Later Planck postulated that the energy of light is proportional to the frequency, and the constant that relates them is known as Planck's constant (h)[1]. His work led to Albert Einstein determining that light exists in discrete quanta of energy, or photons. In 1803, this argument was thought to be put to rest by the mostsimple experiment that ever created, The Double Slit Experiment. Then it was clear to all that the photon has dual nature of particle and wave.

From this point, physics is divided into two parts. One is Classical Physics and the other one is Quantum Physics. Later they saw that the electron has two properties, "Superposition" and 
"Entanglement". And these can be enlightened with the help of Quantum physics rather than Quantum Computing.

Quantum computing began in 1980 when physicist Paul Benioff proposed a quantum mechanical model for the Turing machine. Then in 1981 at MIT the spark of Quantum Computing [15] was stuck by Richard Feynman. Later Richard Feynman and Yuri Manin suggested that a quantum computer had the potential to simulate things a classical computer could not feasibly do.

In 1994, Peter Shor developed his algorithm[14]that allowed quantum computers to successfully process large numbers much faster(exponentially quicker) than the best old algorithm in traditional machines.

From here the journey of Quantum Computer and Quantum Computing has started.

In electrons or photons, they have the property of being able to exist in multiple states is known as Superposition.This means that a single qubit can be described by a linear combination of $\mid 0>$ and $\mid 1>$. It is also called as Mixed State.

It is denoted as $|\Psi>=\alpha| 0>+\beta \mid 1>$

Quantum Entanglement is a physical phenomenon that occurs when a group of particles is produced, interacted, or shared in close proximity in such a way that the quantum state of each particle can be explained without the form of others, including when the particles are separated by a large distance.

A supercomputer has a high level of performance compared to a classical general-purpose computer. We can measure the performance of a supercomputer in floating-point operations per second (FLOPS) instead of million instructions per second (MIPS). Supercomputers contain a large number of processors; they can perform billions and trillions of calculations per second. High-Performance Computer is generally used for higher performance, here we arrange the power of a computer in a way that it can perform much better than a typical classical computer. This type of computer is used for solving larger problems in various fields.

In Quantum Computing, it uses the collective properties of quantum states like Superposition, Entanglement, Interference to perform large calculations in the shortest amount of time. It uses the Qubits in the place of classical bits.

In the case of the quantum circuit model of computation, a quantum logic gate is the most basic quantum circuit which can operate on a small number of qubits. These quantum logic gates are the building blocks of a quantum circuit.

\section{CLASSICAL COMPUTING}

In Classical Computers we can store information as bits, it'll be either 0 or 1 . It is used by large-scale multi-purpose computers and devices. In this type, we have two possible states, 0 or 1 . Here calculations are deterministic. Data processing is done by logic and in a sequential manner.

\section{SUPER COMPUTERS}

As we know there are some limitations of a classical computer so the concept of supercomputer came. A supercomputer is a type of large computer with the resource of multiple computers [10]. It is a very large size computer. These types of computers are expensive to build because here we can't add or remove any components from the supercomputer.

\section{HIGH-PERFORMANCE COMPUTERS}

High-Performance Computer [11] is generally used for higher performance, here we arrange the power of a computer in a way that it can perform much better than a typical classical computer. This type of computer is used for solving larger problems in various fields. Basically, here we combine more than one Super Computer and parallel computing 
techniques to achieve the desired target.HPC is cheaper than supercomputers because we can easily add or remove components here.

\section{INTRODUCTION TO SUPERPOSITION}

let's focus on the two properties of electrons and for the experiment purpose, call them Colour and Hardness. The only observable colours are "black" and "white" and the only observable hardness are "hard" and "soft" (binary properties). These properties can't take any other observable values. So, here we have a colour box and a hardness box which will measure the colour of the electron that is either white or black and the hardness of the electron that is hard or soft.

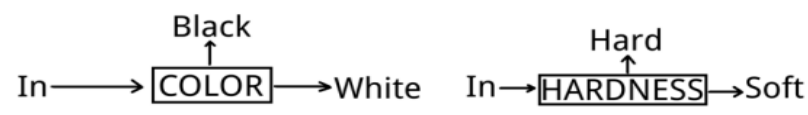

fig1: Colour Box and Hardness Box

This colour box has three apertures, one in port and two out ports (one which sends out white electrons and the other sends out black electrons).

This hardness box has three apertures, one in port and two out ports (one which sends out hard electrons and the other sends out soft electrons).

One key property of these colour boxes and hardness boxes is that they are repeatable.

Now the question is these two properties colour and hardness are correlated? Suppose, we know the colour of the electron is white, so can we say what will be the hardness of the electron? We can answer this question using our boxes and run some experiments. If we send a random pile of an electron into these boxes then the useful thing to know, they come out about 50-50 in a ratio. Now, we are sending a random set of electrons into the colour box and then passing it through the hardness box we can see that $50 \%$ of the white electron comes out from the hardness box as hard electrons and the other $50 \%$ of the white electron comes out from the hardness box as soft electrons.

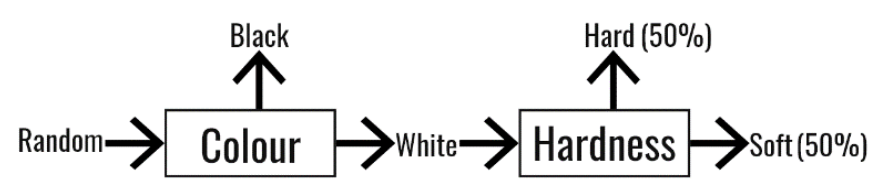

fig2: ratio of hard \& soft electrons

If we interchange the position of two boxes then also, we'll have output with a 50-50 ratio. From this, we can say that knowing the hardness does not give us any information about the colour, and knowing the colour does not give us any information about the hardness. These are independent facts, independent properties. They are not correlated. Now do some more experiments with these boxes. If we add another colour box in the previous system then also, we'll have output with a 50-50 ratio of white electrons and black electrons.

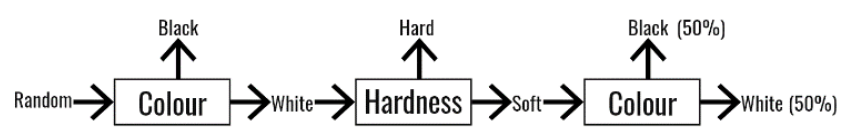

fig3: ratio of black \& white electrons

This is suspicious! Surely, there's some additional property of the electron that we just have not measured yet that determines whether it comes out of the second colour box, black or white. And the interesting fact is no one found such property which can tell which electron will come out from which port. Only we can tell that it's completely random. And as we will see later, using Bell's inequality, we can confirm that such things do not exist. Now, let's take a Hardness box and set two mirrors in the path of the two out port and add a box that will combine the beams together into a single beam and we'll send the output into a hardness box again. We are passing random white electrons into the hardness box. And 
the result of this experiment will be we'll have a 50-

50 ratio of hard and soft electrons.

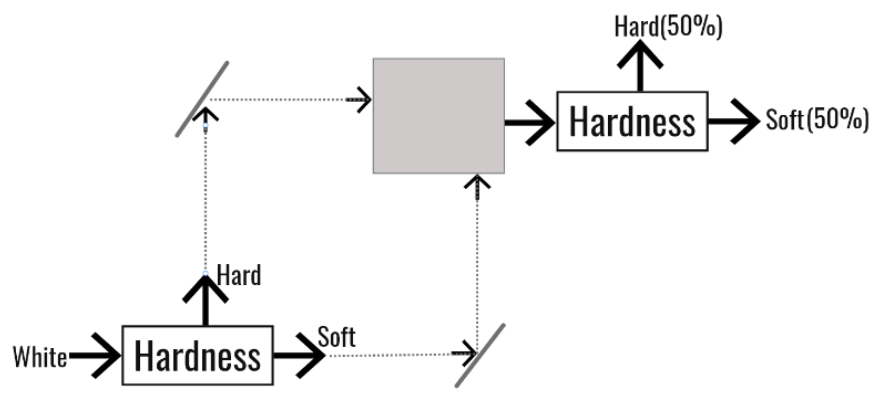

Fig 4: ratio of hard \& soft electrons with mirrors and combination-box

If we do this same experiment with the colour box then also, we will have the same $50-50$ ratio of white colour and black colour. Now, let's take a Hardness box and set two mirrors in the path of the two out port and add a box that will combine the beams together into a single beam and we'll send the output into a colour box. We are passing random white electrons into the hardness box. And the result of this experiment will be we'll have a $0-100$ ratio of black and white electrons.

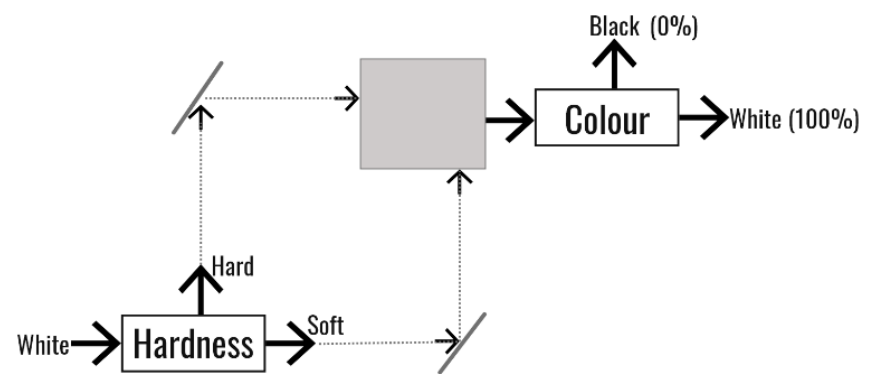

fig5: ratio of black \& white electrons with mirror and combination-box

Now, let's take a Hardness box and set two mirrors in the path of the two out port and add a box that will combine the beams together into a single beam and we'll send the output into a colour box. We are passing random white electrons into the hardness box. But we got a wall on the way of the soft beam so, it'll observe the soft electrons. And the result of this experiment will be we'll have a 50-50 ratio of black and white electrons.

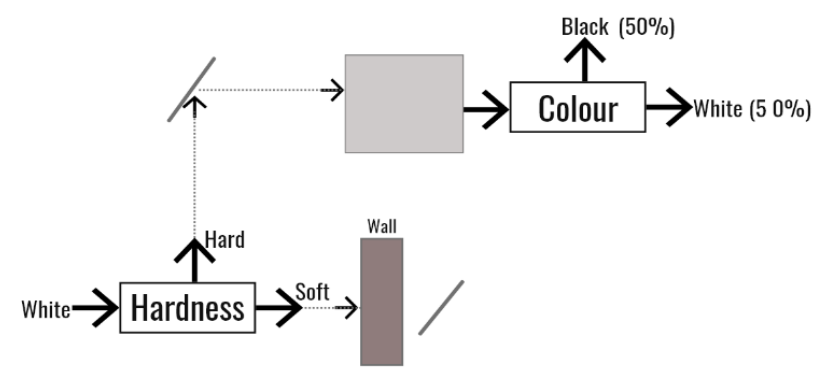

Fig 6: ratio of black \& white electrons with mirror, combination-box and a wall on the path

When the experiment is accurate and the arguments are correct, the electrons actually do something we have never dreamed of before. Electrons have mechanisms or mechanisms that are not in line with what we have discussed so far. This is also true of molecules, viruses, and other microscopic substances, although the results are difficult to see. Physicists call that such thingsis defined by a special property, Superposition [2]. In electrons or photons, they have the property of being able to exist in multiple states is known as Superposition.This means we can represent a single qubit by a linear combination of $\mid 0>$ and $\mid 1>$. It is also called as Mixed State $(|\Psi>=\alpha| 0>+\beta \mid 1$ $>$ ).

\section{INTRODUCTION TO ENTANGLEMENT}

Entanglement [13] comes to play when we have two non-interacting particles.

These two non-interacting particles can have a limited distance or they can have an infinite distance between them. A strong interaction between particles is not required to produce the entanglement property of electrons, these particles can be non-interacting.

Let's assume particle 1 can be in states $\mathrm{u} 1$ and $\mathrm{u} 2$, and particle 2 can be in states $v 1$ and v2. These are the possible states of particle 1 and particle 2 .

particle1 -> |u1> , |u2>

particle2 -> |v1>, |v2> 
Now discuss the quantum state of these two particles. So we know that these two particles are not interacting, We can tell what particle 1 is doing and what particle 2 is doing. First, assume that particle 1 is doing $\mathrm{u} 1$ and particle 2 is doing $\mathrm{v} 1$ and mathematically, we like to make this look like a state, and we want to write it in a coherent way. So, we are going to multiply these two things but we must say sort of multiply because these are vector states, here we put something called a tensor product $[\otimes]$.

So, it'll look like this $->|\mathrm{u} 1>\otimes| \mathrm{v} 1>$, here we don't move things across and this is a possible state.

Now, we could have a different state. Because particle 1 and particle 2 could be doing something different.

particle1 -> $\alpha 1|\mathrm{u} 1>+\alpha 2| \mathrm{u} 2>$

particle2 $->\beta 1|\mathrm{v} 1>+\beta 2| \mathrm{v} 2>$

So, it'll look like this $->\quad(\alpha 1|\mathrm{u} 1>+\alpha 2| \mathrm{u} 2>) \otimes(\beta 1$ $|\mathrm{v} 1>+\beta 2| \mathrm{v} 2>)$

The rules of tensor multiplication to combine those states are just like a product, except you never move the states across. So, from the above tensor operation, we have this result $->$

$\alpha 1 \beta 1|\mathrm{u} 1>\otimes| \mathrm{v} 1>+\alpha 1 \beta 2|\mathrm{u} 1>\otimes| \mathrm{v} 2>+\alpha 2 \beta 1 \mid \mathrm{u} 2>\otimes$ $|\mathrm{v} 1>+\alpha 2 \beta 2| \mathrm{u} 2>\otimes \mid \mathrm{v} 2>$

This the superposition of the state.

Now, if we try to factorize this, $|\mathrm{u} 1>\otimes| \mathrm{v} 1>+\mid \mathrm{u} 2>\otimes$ $\mid \mathrm{v} 2>$,

we'll see a result that this state is un-factorizable. It's a funny state in which you cannot say that this quantum state can be described by telling what the first particle is doing and what the sound particle is doing. Here, what the first particle is doing depends on the second particle and what the second particle is doing depends on the first particle. This is an Entangled state.

If we take two particles with spin, for example, we can build entangled states of two spins $1 / 2$ particles. And this state will look like this ->

$|\uparrow ; \mathrm{z}>1 \otimes| \downarrow ; \mathrm{z}>2+|\downarrow ; \mathrm{z}>1 \otimes| \uparrow ; \mathrm{z}>2$

If we say, Alice has one particle, Bob has the other particle, maybe Alice is on the moon with her electron and Bob is on the earth with his electron and if these two electrons are in this above state then we can say Alice and Bob share an entangled pair. Here, if Bob measures his spin and he finds his spin as a down spin then if Alice also measures her electron spin at that instance of time she will find her spin as an up spin. Einstein objected to this phenomenon and he said this is a spooky behaviour of the electron. But in 1964 John Bell discovered his "Bell Inequalities"[3] that demonstrated that if Alice and Bob can measure in three different directions, they will find correlations that are impossible to explain with classical physics. This can be defined by the quantum physics entanglement theory.

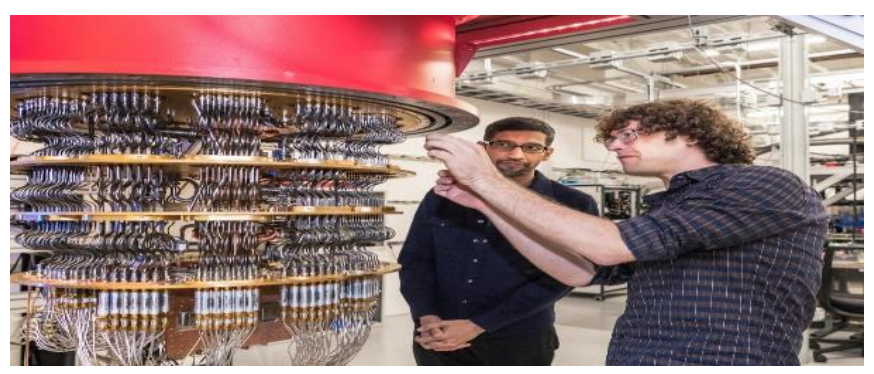

fig7: Google CEO Sundar Pichai with Google Quantum Computer (2020).[18]

\section{THE LINK BETWEEN CLASSICAL COMPUTING AND QUANTUM COMPUTING}

As we know, that classical computer can take a single input, and it'll give us a single output. Basically, classical computers can give us a straightforward answer. This is the main difference between classical and quantum computing because quantum computers work in a different manner, Quantum Computers can take a range of different inputs and can deliver a range of possible answers or outputs.

So, this special type of computing is only useful when we are dealing with complex computational problems, where we have a set of inputs and many complex algorithms. But if we want to do such complex computation in a classical computer then it'll take a very long time to compute all the difficult steps.

Actually, quantum computers can narrow down our possible range of inputs after this process we can have 
a straightforward answer from the classical computers by using that small range of inputs obtained by the quantum computers. In this way, we can marge the ability of the quantum computer and the classical computer. It'll help us now to handle complex computations and other time-consuming tasks.

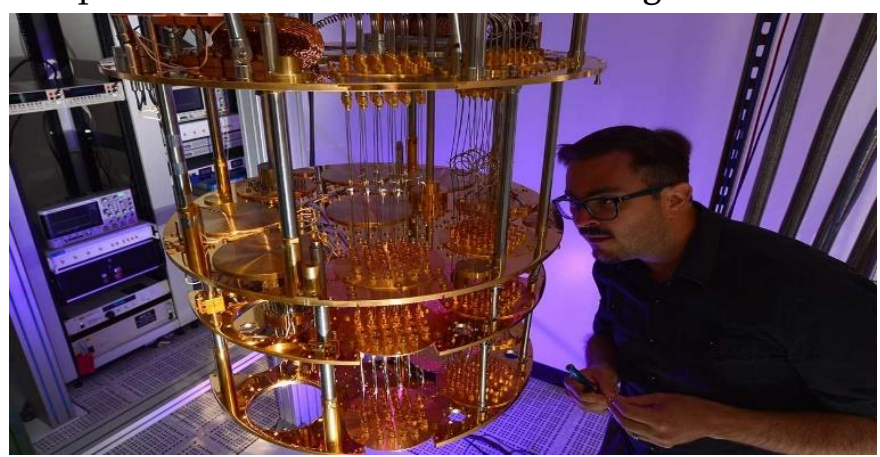

fig8: IBM Director of quantum hardware, Jim Clarke with IBM Quantum Computer.[19]

\section{ADVANTAGES IN THE EVOLUTION OF QUANTUM COMPUTING}

\section{A. Drug Development}

Computer simulation of drug molecules is important as it reduces costs and time, sometimes dramatically. Today, this type of imitation is possible only with tiny molecules. If, however, companies are interested in proteins, which often contain thousands of elements, they need to make and test their properties in real life because modern computer resources are not enough to make accurate simulations. Quantum simulation another hot topic in quantum computing, can reduce the development costs and it'll help bring drugs to the market faster. However, as quantum computing always returns a range of possibilities, the molecular structure of the drug will still need to be verified by a classical computer.

\section{B. Development Problems}

What is the proper installation of equipment in the factory? What is the best way to use vehicles to ensure a successful transport network? What is the best investment strategy for a full 5 years, 10 or 30 years? These are complex issues where the best answer is not always visible. With quantum computers, one can dramatically reduce the possibilities and then use the old computers to get direct answers. These problems abound in various fields, from manufacturing to transportation to finance.

\section{Quantum Artificial Intelligence}

Billions of dollars are invested in private cars. The goal is to make cars smarter to fit on busy roads anywhere in the world. Although now there is lot of talent at work in making AI algorithms to learn and to drive them, but accidents are still a problem. Quantum AI, which may be faster and more powerful than current methods, can help solve this problem. The benefits can only be reaped a decade from now, however, as quantum $\mathrm{AI}$ is developed much less today than quantum simulation or cryptography. Therefore, most AI algorithms will continue to be distributed to older computers. While it is still too early to predict with confidence right now, it is not that most AI will be worth it in a few decades.

\section{Cryptography}

Modern security systems rely on random numbers and numerical measurements that ancient computers can combine to create a password but rarely resolve to break a password. Within a few years, however, quantum computers could become so powerful that they could bypass any secret number. That's why it's important for researchers to start investing in new, more quantum cryptography. Quantum technology is a two-edged sword, with the help of this we can, not only crack the entire password but also be able to generate new one, immovable cryptographic keys. Space is moving fast to embrace this new reality. As old computers will always work, it is important that quantum-safe cryptography is present in this case as well. This is possible, and companies have already begun to protect their data from older computers in this way. At this current time, the QKD (Quantum 
Key Distribution) is the most popular quantum cryptography technique.

\section{RECENT DEVELOPMENTS IN QUANTUM COMPUTING}

Recent advances in quantum supremacy provide evidence that quantum computers are more capable of solving certain problems than any older computer. Many companies like IBM and Google now focusing on their development parts and pushing their efforts to produce superconducting qubits, while educational startups like IonQ focus on ion qubits. Because Superconducting qubits are faster, but on the other hand ion qubits are highly interconnected, require a few steps to perform calculations, and this compensates for slower operating speeds. This term describes the methods of arranging quantum calculations within the quantum error correction code, such as CSS code or verification code. Google, IBM, and a host of different startups hope to double their quantum computing power each year. This means that some companies need to follow in the footsteps of Barclays, BASF, BMW, Dow, and ExxonMobil, which are already operating in quantum technology [4]. In the year 2019, Google's quantum computer made calculations in less than four minutes that would take the world's most powerful computer 10,000 years to make, and 158 million times faster than the World's Fastest Computer. Google's Quantum Computer 158 million Faster Than the Fastest Supercomputer in the World | by Vidar | Guess | Feb 2021 | Medium New Computing in terms of quality, speed, and efficiency continues to gain new ground. Private-public cooperation is increasingly sought after by quantum understanding. Five new National Quantum Information Science Research institutes were created by researchers from the U.S. Department of Energy. from education, U.S. national labs and industries will be working together to help promote the scientific research of quantum knowledge. Companies involved in research institutions include projects IBM, Microsoft, Intel, Applied Materials, and Lockheed Martin. These funds come from the $\$ 1.2$ billion allocated by the National Quantum Initiative Act.

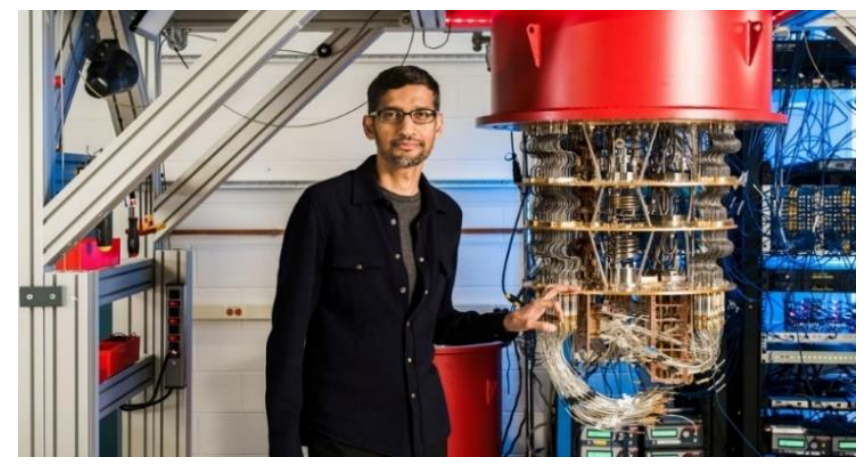

fig 9: Google CEO Sundar Pichai with Google Quantum Computer (2020).[20]

\section{GOOGLE'S QUANTUM JOURNEY [16]}

\section{A. Stepping into the unknown}

We have a lot of ideas, and some proven ideas, about what we can do with a useful quantum computer, which has been fixed by errors. Having said that, no one knows what we will be able to do with a quantum computer once we have built it. One thing we do know: it will change the way we solve problems and what we can develop. Achieving error correction will not be an easy journey, however. As with any good adventure, we are sure to encounter some unexpected obstacles and interesting obstacles along the way.

\section{B. We started here, in the world of classical computing}

Old computers are great. Empower some of mankind's greatest achievements, connecting people around the world with information, ideas, and more. But we are beginning to find the limitations of the problems we can solve in this paradigm. To get beyond this point, we will need quantum computers and older computers that work together. 


\section{1 Qubit}

In 1982, physicist Richard Feynman proposed the idea of building universal quantum computers to mimic other quantum systems. In the 1990s and beyond, scientists proved that we can build computers that can hold quantum information, known as qubits for just a few million seconds. The next challenge for all of us was to find a way to make qubits that could hold data for as long as we wanted - harder than it sounds.

\section{What lies beyond the classical computer?}

Classic computers and quantum computers are used to solve different types of problems. By creating an old computer, we represent what we think of the world as fragments of information in the order of zero and one, and use the mind to make decisions. Is anyone at the door? Yes (1) or no (0)? If so (1); then open the door. To solve easy-to-use problems, we have built computers that use qubits instead of bits. Qubits represent the earth in terms of uncertainty, and we use quantum mechanics to make decisions based on the probability that our qubits are in one (1) or another (0) state. These computers enable us to imitate the way the world works, instead of just thinking about how it works.

\section{E. The rea of NISQ}

In 2019, Google demonstrated that quantum computing is a reality, rather than a speculative one. We have not built portable qubits that can last as long as we like, but we have built them up to last long enough to perform some calculations faster than the world's most powerful computer. At this point, humanity has crossed the line from the old computer to the Noisy computer age, the Intermediate Scale Quantum (NISQ). NISQ computers can perform tasks with minimal reliability but beyond the power of older computers. Although we have limited reliability, we can improve our scientific knowledge in the NISQ era.

\section{F. Chemistry with Quantum Computing}

Chemical reactions are machines that make all living things work, and much more. Everything from plants that convert sunlight into energy stored in a tree that we use to reduce fever is the result of chemical reactions. For the past 100 years, we have been developing mathematical methods for describing chemicals and their interactions. Chemical reactions are most accurately described by quantum mechanics. We have developed computational techniques to describe other chemical reactions, but some of the most important reactions remain far away from older computers. In a recent experiment, we used 12 qubits to mimic a simple chemical reaction on a quantum computer. This was a big step forward.

\section{G. Physics with Quantum Computing}

One thing that sets humans apart from most other living things on earth is our ability to use energy to perform useful tasks. We used fire, then steam, and finally electricity. Our modern way of life is now dependent on electricity for almost all energy.

Using quantum computers, we can study the physics of how electrons interact with different objects in ways that sometimes do not occur in older computers. In a recent study, we used 16 qubits on a quantum computer to show how we could mimic the power of the Fermi-Hubbard model, which predicts how we can create something that loses a small amount of energy as electrons move through it.

\section{H. Graph Theory with Quantum Computing:}

Our luxury, sometimes our lives, depends on a mental branch called Logistics. In Logistics, we learn things like how to successfully move a package from $\mathrm{Da}$ Nang to Dublin, and how many tons of aluminium car manufacturers should order this month. Logistics relies heavily on Graphic theory: a study of how interconnected systems behave. We do not have 
complete computer solutions for most graph problems. However, in our most recent QAOA study, we used 17 qubits to demonstrate that we could successfully develop the graphic problem that is best known for the Sherrington-Kirkpatrick model.

\section{Studying chaos}

Consider the distribution of a drop of ink in a glass of water. Can you predict where the ink will go? Answering this question is key to discovering how chaos occurs in quantum systems and how complexity arises. Recently, we used 53 qubits to learn how quantum entanglement waves can propagate ballistically, depending on the design of our circuit and we can control the speed of entanglement distribution by changing the shape of the circuit.

We have two accepts here-

Removing the physics of out-of-time-order correlators.

Dynamic Information in Complex Quantum Circuits.

\section{J. Beyond the performance of classical computing}

Google's Sycamore quantum processor was able to do a job in 200 seconds that could take 10,000 years on an old computer. We set up a quantum computer and the world's most powerful supercomputer (at the time) to select random $0 \mathrm{~s}$ and $1 \mathrm{~s}$ randomly, and to measure how random it was. This may not seem to be very useful, but computing is a difficult computer task. It was the first time that any quantum computer had successfully reached this milestone.

\section{K. Towards a Quantum Computing Future}

By working with universities and research institutes, Google is making an important contribution to ideas and algorithms that we can use for computercorrected computers when we get there.

They will continue to discover new, increasingly complex problems that can be successfully tested by our computers in quantum NISQ computers over older computers.

\section{In search of an eternal qubit}

When it comes to creating a faulty quantum computer and testing its many operating systems, all the new ideas and ideas help us move forward. So, whether someone is a beginner in quantum computing or a researcher working on high-level problems, we want to make it easier for everyone to learn and participate. That's why we created Cirq, the open-source Python Library for quantum computer programming in the modern NISQ era.

\section{QUANTUM COMPUTING DEVELOPMENTS IN INDIA}

India has reached an important milestone in its history of research and development. The Ministry of Electronics and Information Technology (MeitY) has released the long-awaited quantum imitation toolbox [7]. This is also the first 'Quantum Computer Simulator (QSim) Toolkit' in the country. According to the report, IISc Bangalore, IIT Roorkee, and CDAC have teamed up for the first time in India to address a shared issue of expanding the boundaries of quantum computing research nationwide [8]. The great advantage is that researchers and students will be able to perform quantum computing research effectively using this device.

The toolkit will enable researchers and students to study quantum computing effectively. The QSim project - jointly developed by IIT Roorkee, IISc Bangalore, and C-DAC - will help develop and refine quantum algorithms.

Significantly, quantum simulators are devices that allow people to learn quantum effects, which are difficult to learn in the lab. IIT-Roorkee [9] officials said that in areas such as cryptography, computational chemistry, and machine learning, quantum computing promises a significant increase in computer power. The Premier's Office added that QSim is a first-of-its-kind 'traditional' tool kit and is intended to be an important tool for learning and 
understanding the realities of editing using quantum computers.

"The quantum simulator launched today (August 27) is poised to be a key enabler in the direction of quantum education and research," said

Professor AK Chaturvedi, director of IIT-Roorkee.

\section{EXPERIMENT}

In the picture below, we have the $\mathrm{X}$-axis, which represents left to right time increases and we have $\mathrm{Y}$ axis which has the Qubit index. So, the author took 5 Qubits for both experiments, and also the author took 5 classical bits to see the measurement at the end.

In the first one, the author places a Hadamard Gate in Qubit number 1 which is named q0, by which the author creates a superposition of Quantum states. Also, the author places a measurement in q0, by which they can see the result of the experiment. As result, they have got a probability of $0 \mathrm{~s}$ and $1 \mathrm{~s}$ in a ratio of 50-50 percentage.

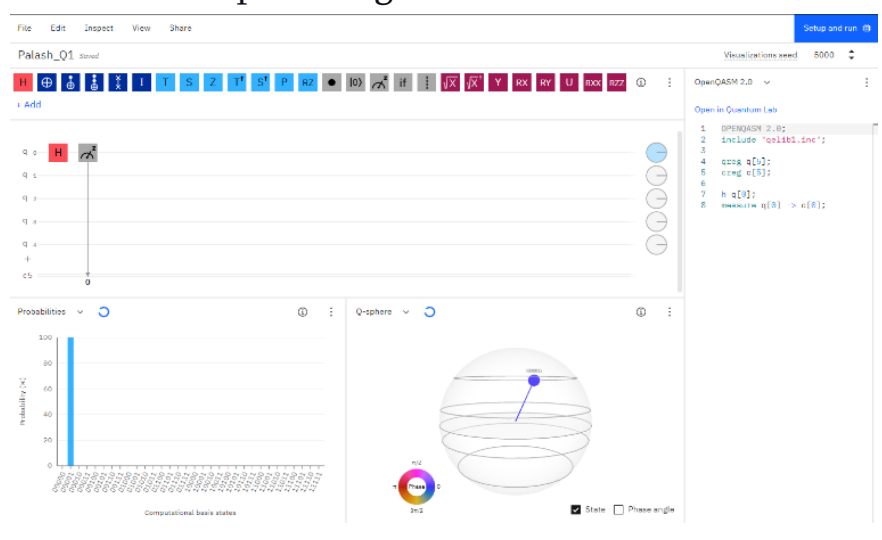

Experiment - 1 with IBM Quantum Computer (2021)

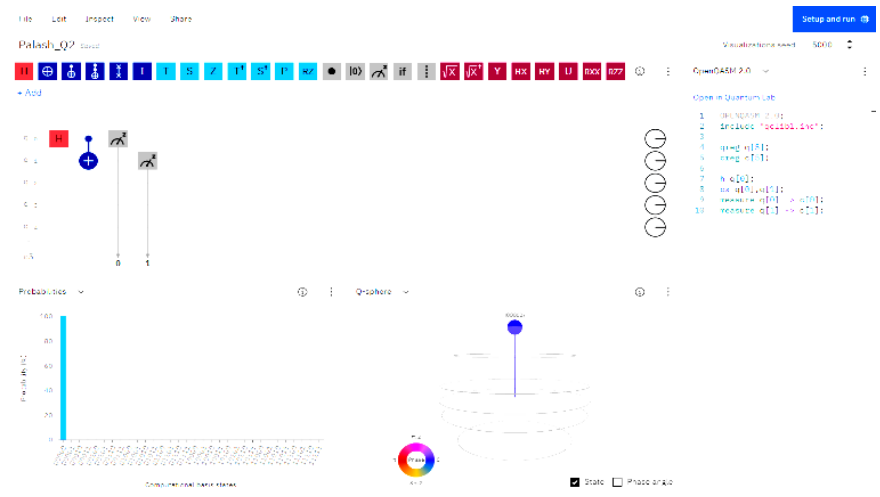

Experiment - 2 with IBM Quantum Computer (2021)
In the second one, the author places a Hadamard Gate in Qubit number 1 which is named q0, by which the author creates a superposition of Quantum states. Then the author uses a CNOT Gate, where q0 is the control Qubit, and q1 is the target Qubit. By this, they are achieving The Bell states. Now they place a measurement in q0, and another measurement in q1 so that the authors can have the result more clearly as classical bits.

\section{LIMITATIONS}

(i) Qubits can be operated at 20 millikelvins ( $-273^{\circ}$ Celsius). So, it's hard to maintain such a temperature, and we can't be able to operate a Quantum Computer at our room temperature.

(ii) Quantum Algorithms are hard to understand and programming for a quantum computer is a difficult job to be done. Although there is a Python library by the Google Team named Cirq, is very useful to perform quantum computing programs. (Cirq [17]is a Python software library for writing, manipulating, and optimizing quantum circuits, and then running them on quantum computers and quantum simulators.)

(iii) Quantum computers are very difficult to make, build and organize. As a result, they are crippled by sound errors, errors, and loss of quantum compliance, which are important in their performance but yet disintegrate before any irrational system has a chance to run until it is completed.

(iv) Qubits can't store data or a set of data for a long time, so this is another problem with quantum computers nowadays.

\section{CONCLUTION AND FUTURE SCOPES}

In the near future - perhaps within 5 to 10 years, but who knows, maybe a lot sooner - quantum computing will be at a stage where it can be used to solve problems that improve our lives, on an everyday 
basis - Intel's head of quantum research, Jim Clarke, calls this "quantum practicality.[5]"Quantum computers won't be able to replace classical computers for now but in the case of the near future maybe it'll be possible to replace classical computers with quantum computers. This is kind of similar to older computers, such as the mainframes created by IBM in the mid-20th century, which did not become operational for everyday use until universal operating systems and programming languages were discovered. Currently, quantum computer control systems fill a small room - this efficiency reduces it to a single chip in the near future. Once this value has been proven, experts hope that quantum computers will be used to build systems that help us cope with climate change. One of the ways in which this can be done is to create new types of agricultural fertilizers. Switching to new compound fertilizers can reduce the earth's natural gas consumption by 3 to 5 percent. This will be done by building new auxiliary molecules that are more efficient at creating the necessary chemicals. Quantum computers also have a huge impact on machine learning and learning. These computerassisted computer systems - which include learning programs and improvements in their operations operate using large neural networks, requiring high computer computing. Quantum-powered AI will provide us with devices that can think and learn faster than before. This will enable more advanced systems to be replicated and modelled. Imitation is based on our understanding of reality, in order to replicate the rules within our models. This means understanding the behaviours of an object and the quantum level. Richard Feynman[6], a Nobel Prizewinning physicist who has helped to explain much of what we know about quantum, argued that only quantum computers could be powerful enough to accurately measure quantum function.If this were possible (and would require quantum computers much more powerful than the ones we have today, instead of thousands of qubits), we should be able to build models that accurately replicate the most sophisticated systems that can be modelled today such. such as electromagnetic radiation, gravity, and perhaps even the biological brain. Whatever the case, it is clear that quantum computing is an exciting field of technological advancement, and we can expect to see it grow and impact our lives over the years perhaps in as important a way as the advent of computers in the last century, and growth of the Internet in this century.Quantum Computing won't be able to consume the world of computing but surely enough will have a major impact on the computing universe for the next couple of decades and will maintain co-relation with classical computing.

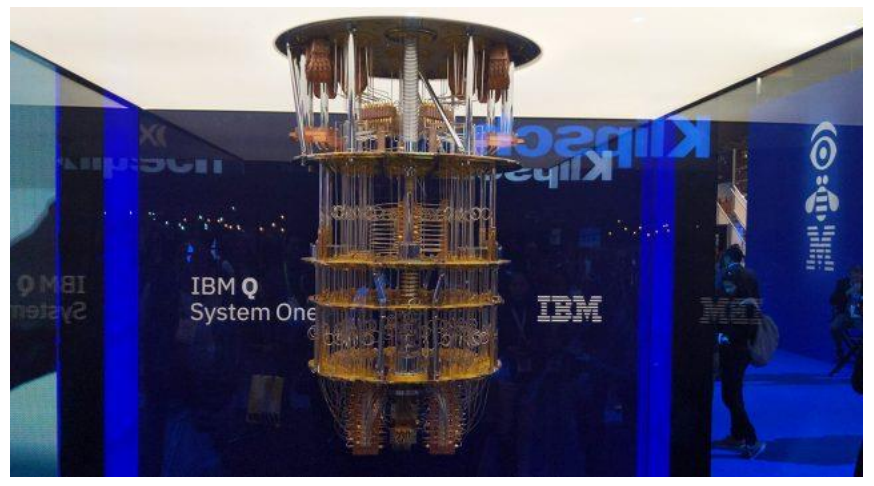

IBM Q System One (2021) [21]

\section{REFERENCES}

[1]. Lumen, Planck's Quantum Theory, https://courses.lumenlearning.com/introchem/c hapter/plancks-quantumtheory/\#: : :text=Planck\%20postulated\%20that\% 20the\%20energy,quanta\%20of\%20energy\%2C \%20or\%20photons.

[2]. Allan Adams, Introduction to Superposition, June 19, 2014, https://youtu.be/lZ3bPUKo5zc

[3]. BARTON ZWIEBACH, Entanglement, July 5, 2017, https://ocw.mit.edu/courses/physics/8-04quantum-physics-i-spring-2016/videolectures/part-1/entanglement/

[4]. Ari Joury, Will quantum computers replace their classical counterparts? February 1, 2021, https://towardsdatascience.com/will-quantum- 
computers-replace-their-classical-counterparts847e20e32fc2

[5]. Bernard Marr, The Future of Quantum Computing, August 9, 2021, https://bernardmarr.com/the-future-ofquantum-computing/

[6]. Bernard Marr, The Future of Quantum Computing, August 9, 2021, https://bernardmarr.com/the-future-ofquantum-computing/

[7]. Tapan Susheel, India's first Quantum Computer Simulator toolkit launched, August 28, 2021, https://timesofindia.indiatimes.com/city/dehrad un/indias-first-quantum-computer-simulatortoolkit-launched/articleshow/85696357.cms

[8]. Tapan Susheel, India's first Quantum Computer Simulator toolkit launched, August 28, 2021, https://timesofindia.indiatimes.com/city/dehrad un/indias-first-quantum-computer-simulatortoolkit-launched/articleshow/85696357.cms

[9]. Tapan Susheel, India's first Quantum Computer Simulator toolkit launched, August 28, 2021, https://timesofindia.indiatimes.com/city/dehrad un/indias-first-quantum-computer-simulatortoolkit-launched/articleshow/85696357.cms

[10]. William L. Hosch, supercomputer, November 28 , 2019,

https://www.britannica.com/technology/superc omputer

[11]. NetApp, What is high-performance computing?, 2021, https://www.netapp.com/data-storage/highperformance-computing/what-is-hpc/

[12]. TechTarget, classical computing, 2021, https://whatis.techtarget.com/definition/classica l-computing

[13]. Barton Zwiebach, Entanglement, July 5, 2017, https://youtu.be/G3HSP3qMgKI

[14]. IBM Quantum Composer, Shor's algorithm, 2021, https://quantumcomputing.ibm.com/composer/docs/iqx/guide/s hors-algorithm
[15]. Microsoft, Introduction to quantum computing, 2021, https://azure.microsoft.com/enus/overview/what-is-quantumcomputing/\#how-it-works

[16]. Quantum AI, Our quantum computing journey, 2021, https://quantumai.google/learn/map

[17]. QuantumAI website, 2021, https:/quantumai.google/cirq

[18]. Google CEO Sundar Pichai with Google Quantum Computer Picture, 2020,https://ichef.bbci.co.uk/news/976/cpsprod pb/107C3/production/_119332576_qc.png

[19]. IBM Director of quantum hardware, Jim Clarke with IBM Quantum Computer, 2021, https://spectrum.ieee.org/media-library/intelquantum-computing-researcher-davidmichalak-inspects-part-of-a-quantumcomputing-system-at-the-companys-hillsboroore-campus.jpg?id=25592299

[20]. Google CEO Sundar Pichai with Google Quantum Computer Picture, 2020,https://media.wired.com/photos/5e333e15 be05400008f39506/1:1/w_920,h_920,c_limit/Bu siness-QuantumComputing-Google-

L1001386.jpg

[21]. IBM Q System One Picture, 2021, https://www.extremetech.com/wpcontent/uploads/2019/01/IBM-Q-System-Onedisplay-640x353.jpg

\section{AUTHOR PROFILE}

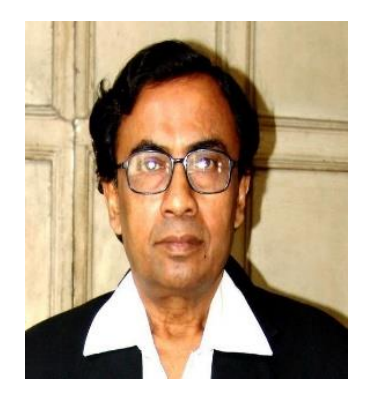

Dr. Asoke Nath is working as Associate Professor in the Department of Computer Science, St. Xavier's College (Autonomous), Kolkata. He is engaged in research work in the field of Cryptography and Network Security, Steganography, Green Computing, Big data analytics, Li-Fi Technology, Mathematical modelling of Social 
Area Networks, MOOCs, Quantum Computing etc. He has published more than 257 research articles in different Journals and conference proceedings.

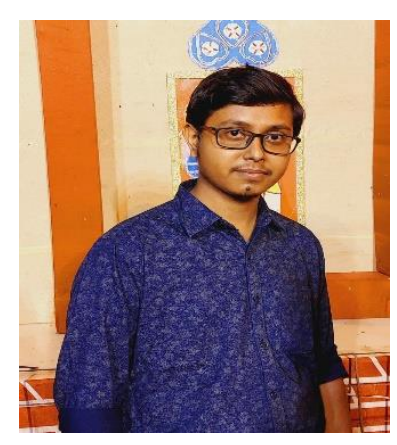

Mr. Palash Dutta Banik is a student of St. Xavier's College, currently pursuing M.Sc. in Computer Science. His interests lie in the field of Quantum Computing, Machine Learning, Coding, App Development, UI

Design, Cyber Security, AI and real-world project implementation of these fields.

\section{Cite this article as :}

Palash Dutta Banik, Asoke Nath, "Past, Present and Future of Quantum Computing: A Systematic Study", International Journal of Scientific Research in Computer Science, Engineering and Information Technology (IJSRCSEIT), ISSN : 2456-3307, Volume 7 Issue 6, pp. 351-363, November-December 2021. Available at

doi $\quad$ : https://doi.org/10.32628/CSEIT217694

Journal URL : https://ijsrcseit.com/CSEIT217694 